\title{
Avaliação da atividade moluscicida do látex de três espécies de Euphorbia (Euphorbiaceae) sobre Leptinaria unilamellata D’Orbigny, 1835 (Gastropoda - Subulinidae)
}

\author{
AFONSO-NETO, I.S. ${ }^{1}$; BESSA, E.A. ${ }^{2}$; SOARES, G.L.G ${ }^{3}$ \\ ${ }^{1}$ Universidade Presidente Antônio Carlos, UNIPAC - Campus VI, Av. Juiz de Fora, 1100 - Granjas Bethânia, CEP: \\ 36.048-000, Juiz de Fora-Brasil e-mail: inesaneto@yahoo.com.br ${ }^{2}$ PPG Ciências Biológicas - Comportamento e \\ Biologia Animal, Universidade Federal de Juiz de Fora, CEP: 36.330-900, Juiz de Fora-Brasil ${ }^{3}$ Departamento de \\ Botânica, Instituto de Ciências Biológicas, Universidade Federal do Rio Grande do Sul, CEP: 90.501-970, Porto \\ Alegre-Brasil
}

\begin{abstract}
RESUMO: Gastrópodes pulmonados terrestres podem atuar como hospedeiros intermediários de helmintos. Os primeiros registros do controle químico desses invertebrados datam do início do século XX e as substâncias utilizadas eram toxinas inespecíficas já empregadas no controle de outras pragas. Moluscicidas sintéticos apresentam limitações técnicas que estimularam a busca de substitutos naturais. Dentre as várias espécies vegetais com atividade moluscicida, Euphorbia cotinifolia L., Euphorbia milii des Moul. var. splendens (Bojer ex Hook) Ursch \& Leandri e Euphorbia tirucalli L. despertam atenção pelos excelentes resultados obtidos sobre moluscos aquáticos. Contudo, estudos sobre a atividade de plantas moluscicidas em moluscos terrestres são pouco comuns, apesar de sua grande importância parasitológica e agrícola. As semelhanças anatomo-fisiológicas entre espécies de moluscos aquáticos e terrestres sugerem que estratégias de controle químico possam ter eficiência semelhante para os dois grupos de invertebrados. Com base nessa hipótese, o presente trabalho teve como objetivo avaliar a atividade moluscicida do látex de três espécies do gênero Euphorbia sobre Leptinaria unilamellata dOrbigny, 1835, gastrópode terrestre descrito como hospedeiro intermediário de helmintos trematódeos digenéticos que parasitam animais domésticos. Destas $E$. milii var. splendens apresentou efeito moluscicida elevado sobre $L$. unilamellata, $100 \%$ até uma diluição de $1: 800$, já nos primeiros minutos após a aplicação. Embora citadas na literatura como tóxicas para moluscos aquáticos, E. cotinifolia e E. tirucalli não exibiram atividade moluscicida sobre $L$. unilamellata. Os resultados do presente estudo indicam que o látex de $E$. milii var. splendens pode se constituir em uma estratégia viável de controle químico de moluscos terrestres.
\end{abstract}

Palavras-chave: Leptinaria unilamellata, gastrópodes terrestres, moluscicida, Euphorbia sp.

\begin{abstract}
Assessment of moluscicidal activity of the latex of three Euphorbia (Euphorbiaceae) species on Leptinaria unilamellata D'Obigny 1835 (Gastropoda Subulinidae). Pulmonate terrestrial gastropods can be intermediate hosts for helminthes. The first records of chemically controlling these invertebrates date back to the beginning of the $20^{\text {th }}$ century. The substances used were unspecific toxins which were already used for controlling other pests. Synthetic molluscicides have technical limitations that stimulated a search for natural substitutes. Among the many vegetal species that have molluscicidal activity, Euphorbia cotinifolia L., Euphorbia milii des Moul. var. splendens (Bojer ex Hook) Ursch \& Leandri and Euphorbia tirucalli $\mathrm{L}$. call attention because of the excellent results that have been obtained when they are used on aquatic mollusks. However, studies on the activity of molluscicidal plants on terrestrial mollusks are rare, in spite of its great importance in controlling parasites and to agriculture. Anatomical and physiological similarities among species of aquatic and terrestrial mollusks suggest that chemical control strategies can be effective on both groups of invertebrates. Based on this hypothesis, we assessed the molluscicidal activity of the latex of three species of the genus
\end{abstract}

Recebido para publicação em 20/11/2007

Aceito para publicação em 03/11/2009

Rev. Bras. PI. Med., Botucatu, v.12, n.1, p.90-95, 2010. 
Euphorbia on the terrestrial gastropod Leptinaria unilamellata dOrbigny, 1835, described as an intermediate host of digenetic trematode helminths that parasitize domestic animals. E. miliivar. splendens had the highest molluscicidal effect on L. unilamellata, being $100 \%$ lethal in concentrations up to $1: 1800$ in the first minutes after application. Although $E$. cotinifolia and $E$. tirucalli have been cited in the literature as toxic to aquatic mollusks, they did not have molluscicidal activity on $L$. unilamellata. The results of our study indicate that the latex of $E$. miliivar. splendens may be a viable strategy for the chemical control of terrestrial mollusks.

Key words: Leptinaria unilamellata, terrestrial gastropodes, molluscicide, Euphorbia sp.

\section{INTRODUÇÃO}

Moluscos da classe Gastropoda, tanto aquáticos quanto terrestres, convivem em ambientes onde se encontram comunidades humanas ocupando diferentes habitats e sua relação ecológica com helmintos bem como seus hábitos alimentares podem trazer perturbações à qualidade de vida do homem (Dutra, 1988; Fretter \& Peake, 1978; Jurberg et al., 1989; Raut \& Panigrahi, 1990; Thomas, 1995; Yuan, 1995; Sturrock, 2001).

Há íntima interdependência de helmintos trematódeos digenéticos e gastrópodes pulmonados terrestres e aquáticos, pois esses helmintos utilizamnos como hospedeiros intermediários podendo, devido à existência dessa interação, tornarem-se transmissores de doenças ao homem (Brown, 1978; Yuan, 1995). Logo órgãos de saúde coletiva preconizam o controle desses moluscos (Jurberg, 1987; El-Khoby, 1995; Sturrock, 2001).

Esse controle tem sido realizado há várias décadas com substâncias químicas de origem sintética entre inespecíficas e específicas (Paulini, 1959; Malek \& Cheng, 1974; Michelson, 1987; Sturrock, 1995). Nenhuma dessas substâncias sintéticas é livre de efeitos indesejáveis como: toxidez a espécies simpátricas, outros invertebrados e vertebrados, além de efeito residual no ambiente e toxidez aos humanos (Maleck \& Cheng, 1974; Panigrahi \& Raut, 1993; Sturrock, 1995; Sarquis et al., 1997).

Atualmente há intensificação na busca de produtos naturais de origem vegetal com diferentes atividades biocidas; pois tais substâncias constituem alternativa viável para os produtos sintéticos por preencherem requisitos como biodegradabilidade, menor impacto ambiental e menor custo. Também possuem aceitação por sociedades onde produtos sintéticos, importados de áreas do mundo muito diferentes culturalmente, são mal vistos e consequentemente mal aplicados (Marston \& Hostettman, 1985; Sturrock, 1995; Clark et al., 1997).

Existem muitas espécies de plantas tropicais que possuem substâncias com atividade moluscicida, principalmente entre as famílias Asteraceae, Euphorbiaceae, Fabaceae e Phytolacaceae que contém diferentes tipos de substâncias do metabolismo secundário vegetal que podem ter ação moluscicida (Marston \& Hostettman, 1985; Liu et al., 1997; Alfonso et al., 2000; Rug \& Ruppel, 2000; AlZanbagi et al., 2001).

Na família Euphorbiaceae, plantas lactescentes ricas em terpenóides com representantes nativos e exóticos (Zani et al., 1993; Mabberley, 1997; Oliveira \& Akisue, 1997), o gênero Euphorbia teve os látices de algumas espécies testados em formulações e concentrações variadas apresentando atividade moluscicida excepcional em gastrópodes pulmonados aquáticos (Pereira et al., 1978; Al- Zanbagi et al., 2001).

Poucas são as pesquisas à respeito de controle de moluscos gastrópodes terrestres, especialmente com extratos vegetais. Não existe na literatura especializada citação sobre o controle de Leptinaria unilamellata d'Orbigny, 1835, subulinídeo de distribuição pantropical que é hospedeiro intermediário de helmintos patogênicos para espécies de aves e de mamíferos (Alicata, 1940; Duarte, 1980; Dutra, 1988).

Com base no histórico do uso de plantas da família Euphorbiaceae como moluscicidas (Jurberg et al., 1989) torna-se relevante a determinação da ação dos seus látices sobre espécies de moluscos terrestres. O presente trabalho visa testar a atividade moluscicida dos látices de três espécies do gênero Euphorbia [Euphorbia cotinifolia L., Euphorbia milii des Moul. var. splendens (Bojer ex Hook) Ursch \& Leandri e Euphorbia tirucalli L.] sobre Leptinaria unilamellata d'Orbigny, 1835 em condições de laboratório.

\section{MATERIAL E MÉTODO}

Os testes de atividade moluscicida foram realizados no Laboratório de Moluscos do prédio da Pós-Graduação em Ciências Biológicas/ Comportamento e Biologia Animal (ICB/UFJF) com temperatura ambiente entre 21 e $25^{\circ} \mathrm{C}$, umidade relativa do ar entre 75 e $90 \%$. As medidas de temperatura e umidade foram realizadas com

Rev. Bras. Pl. Med., Botucatu, v.12, n.1, p.90-95, 2010. 
termômetro de máxima e mínima e higrômetro, ambos da marca Incoterm ${ }^{\circledR}$.

A metodologia para avaliação da atividade moluscicida sobre moluscos terrestres foi adaptada a partir das diretrizes sugeridas pela OMS (WHO, 1965) para ensaios com moluscos aquáticos. As adaptações metodológicas visaram contornar as peculiaridades do modelo estudado, principalmente o hábito terrestre do molusco e a natureza do material vegetal testado.

Os moluscos utilizados no presente trabalho foram obtidos da criação matriz do Laboratório de Moluscos, instalado no prédio da Pós-Graduação em Ciências Biológicas/Comportamento e BiologiaAnimal (ICB/UFJF), estabelecida com exemplares de $L$. unilamellata coletados em hortas e jardins no município de Juiz de Fora, MG.

As matrizes foram mantidas em terrários constituídos por uma caixa de cimento amianto com $30 \mathrm{~cm}$ de largura, $30 \mathrm{~cm}$ de comprimento por 16,5 $\mathrm{cm}$ de profundidade. Os terrários foram preenchidos com terra vegetal comercial esterilizada por uma hora a $120^{\circ} \mathrm{C}$ e cobertas com tela de nylon com malha de $1 \mathrm{~mm}$. Diariamente a terra foi umedecida com água e o alimento dos moluscos foi renovado (Bessa \& Araújo, 1995). A ração oferecida aos animais foi preparada no laboratório. Essa ração constitui-se de mistura formada por ração para aves de corte suplementada com carbonato de cálcio, na proporção de 3:1, segundo metodologia descrita por Oliveira et al. (1968). Os animais jovens foram separados da criação matriz logo após o nascimento e criados nas mesmas condições descritas.

As plantas utilizadas para os testes foram coletadas no Campus da UFJF, observando-se sempre o mesmo local de coleta para cada espécie. As amostras de E. milii var. splendens E. cotinifolia foram coletadas em canteiros próximos ao Instituto de Ciências Biológicas, onde são cultivadas como ornamentais. O espécime de $E$. tirucalli testado foi selecionado no Horto de Plantas Medicinais da Faculdade de Farmácia da UFJF. Exemplares dessas três espécies encontram-se registrados no Herbário Leopoldo Krieger (CESJ) sob os seguintes números: Euphorbia splendens Bojer [= Euphorbia milii des Moul var. splendens (Bojer ex Hook) Ursch \& Leandri], o 19804; Euphorbia cotinifolia L., no 30758 e Euphorbia tirucalli L., № 22847.

Os látices foram extraídos pouco antes da realização dos testes a partir de incisões no caule ou nos pecíolos foliares, no caso de E. cotinifolia. $O$ látex obtido foi imediatamente diluído em água na proporção de $1 \mathrm{~mL}$ de látex para $9 \mathrm{~mL}$ de água. Os recipientes com látex diluído foram envoltos em papel alumínio e transportados para o laboratório, onde foram feitas as demais diluições utilizadas. As amostras de látex foram aplicadas imediatamente após 0 preparo, evitando-se ao máximo a sua polimerização. As diferentes diluições dos látices foram aplicadas com borrifador manual de polietileno nos animais e a verificação de mortalidade foi realizada em até 24 horas após o tratamento, seguindo metodologia da literatura (Vasconcellos \& Schall, 1986) adaptada às peculiaridades desse trabalho.

Os testes foram realizados separadamente para cada espécie de Euphorbia em terrários de polietileno com diâmetro superior de $15 \mathrm{~cm}$ e diâmetro inferior de $11 \mathrm{~cm}$ e $9 \mathrm{~cm}$ de altura. Esses terrários foram preenchidos com terra vegetal até a altura de aproximadamente três centímetros e cobertos com escaline branco. Os grupos tratados e os controles foram constituídos por duas repetições de dez animais. Foi aplicado um volume de látex diluído ou de água destilada suficiente para umedecer as paredes e o substrato dos terrários (cerca de $5 \mathrm{~mL}$ ). O grupo controle compreendeu a utilização de água destilada borrifada.

Cada espécie de Euphorbia foi testada num ensaio preliminar, que seguiu o delineamento acima citado, com três diluições do látex recém coletado 1:10, 1:100, 1:1000 (látex: volume final da suspensão em água destilada). Um ensaio subsequente foi utilizado para a análise mais detalhada da toxidez de E. milii splendens, para essas avaliações foram testadas adicionalmente as diluições de 1:200, 1:400, $1: 600$ e 1:800.

Os resultados da atividade moluscicida foram expressos como valores médios ( \pm erro padrão).

Foi elaborada a análise da relação dose/ resposta do látex de $E$. milii splendens e também o cálculo da concentração letal padrão $\left(\mathrm{DL}_{50}\right)$, a partir dos resultados da ação das diluições adicionalmente testadas: 1:200, 1:400, 1:600 e 1:800.

A estimativa das doses letais $\left(\mathrm{DL}_{50}\right)$ para os testes de atividade moluscicida de $E$. milii foi realizado através da regressão logarítmica da relação entre os valores de mortalidade em porcentagem e as doses testadas, convertidas para $\mu \mathrm{L} \mathrm{mL}^{-1}$. Os cálculos e gráficos foram elaborados usando-se o programa Excel para Windows ${ }^{\circledR}$ versão 2000.

\section{RESULTADO}

No primeiro teste de atividade moluscicida dos látices diluídos das três espécies de Euphorbiaceae (Figura 1) observa-se que apenas $E$. milii foi letal para $L$. unilamellata. Nas diluições de $1: 10$ e 1:100 esse látex provocou $100 \%$ de mortalidade dos animais testados num prazo de $24 \mathrm{~h}$. $O$ efeito do látex de $E$. milii diminuiu drasticamente quando diluído a 1:1.000, mas ainda se observou mortalidade de $25 \%$.

Os resultados do segundo ensaio de atividade moluscicida de $E$. milii confirmaram a 
elevada atividade tóxica. Essa espécie mostrou-se $100 \%$ letal para $L$. unilamellata nas diluições de 1:10 a 1:800 (Figura 2). Mais uma vez observou-se queda abrupta da mortalidade para as diluições de látex acima de 1:1.000.

O efeito tóxico do látex de $E$. milii em todas as concentrações já se fazia notar imediatamente após a aplicação. Os animais dos grupos tratados expunham totalmente a massa cefalopodal,

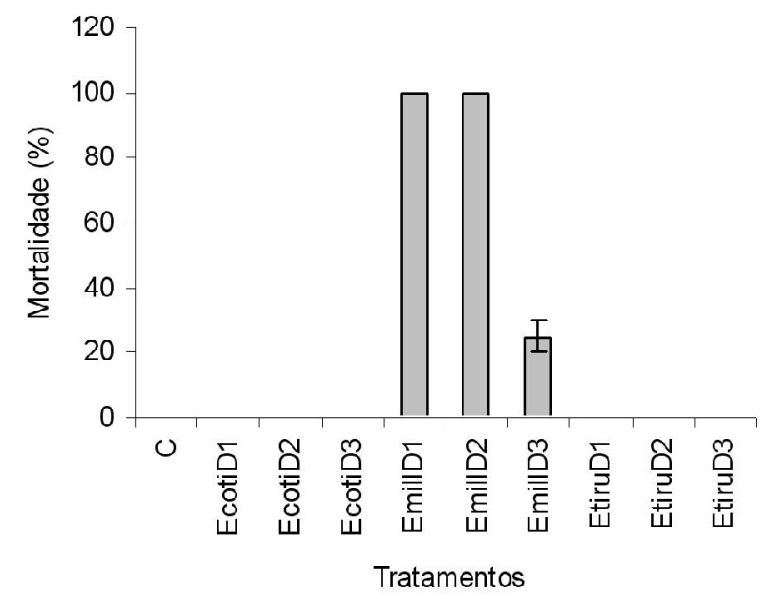

FIGURA 1. Resultado do teste preliminar de atividade moluscicida do látex diluído de três espécies de Euphorbia sobre Leptinaria unilamellata. Legenda C: controle (água destilada); Ecoti: Euphorbia cotinifolia; Emill: Euphorbia miliivar. splendens; Etiru: Euphorbia tirucalli; D1: diluição 1:10; D2: diluição 1:100; D3: diluição 1:1000. apresentavam contrações e intensa secreção de muco. A morte da maioria dos animais podia ser observada nos primeiros 3 minutos e as percentagens máximas de mortalidade em um prazo de $24 \mathrm{~h}$ a partir do início do teste.

Com base nos resultados do segundo teste moluscicida do látex de $E$. milii, pode-se estimar uma $D_{50}=0,52 \mu \mathrm{LL}^{-1}$ desse produto natural sobre $L$. unilamellata (Figura 3).

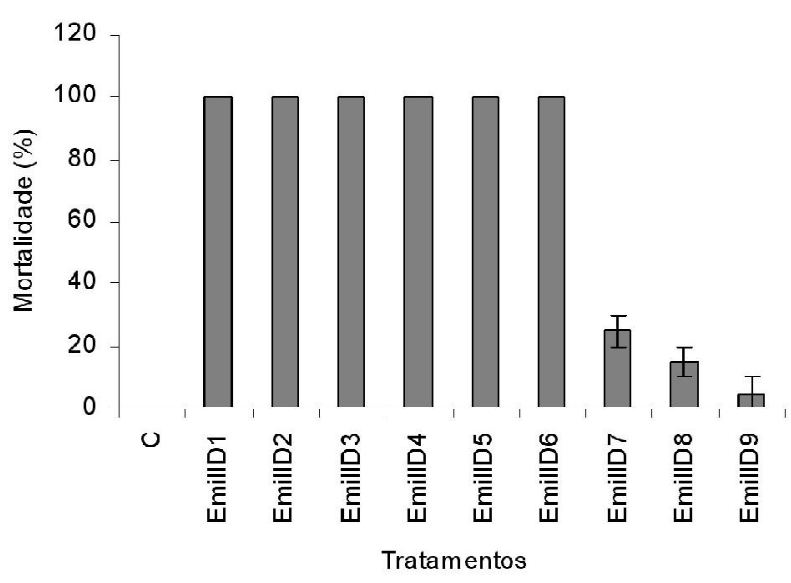

FIGURA 2. Resultado do teste de atividade moluscicida do látex diluído de Euphorbia milii sobre Leptinaria unilamellata. Legenda: C: controle (água destilada); Emill: Euphorbia milii var. splendens; D1:diluição 1:10; D2: diluição 1:100; D3: diluição 1:200; D4: 1:400; D5: 1:600; D6:1:800; D7: 1:1000; D8: 1:10000; D9: 1:100.000.

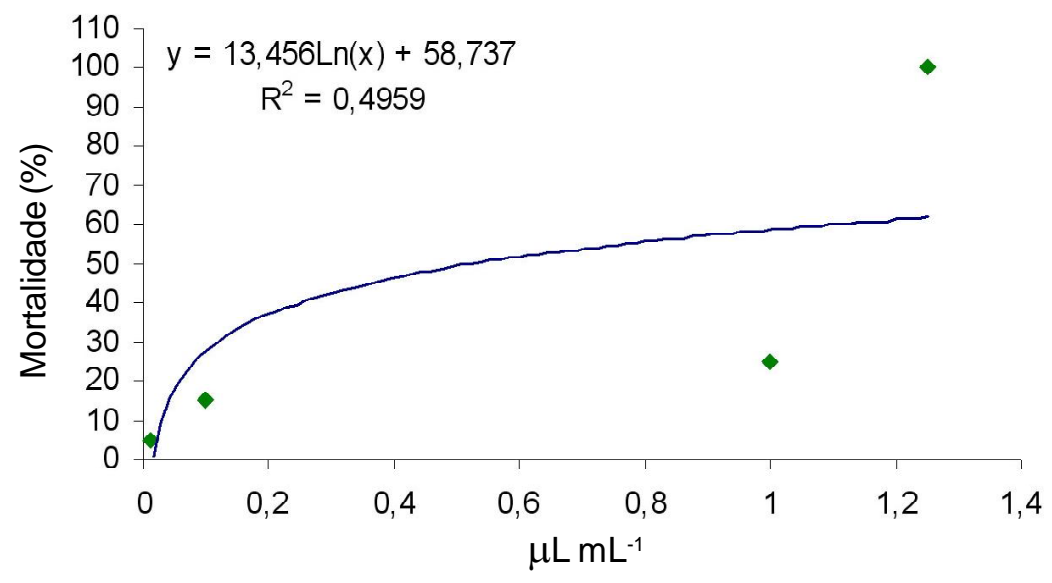

FIGURA 3. Curva dose-resposta da atividade moluscicida de Euphorbia milii sobre Leptinaria unilamellata. Obs: As diluições testadas (Figura 2) foram convertidas para $\mu \mathrm{LLL}^{-1}$. $\mathrm{DL}_{50}=13,456 \mathrm{Ln}(\mathrm{x})+58,737 \backslash \mathrm{DL}_{50}=0,52 \mu \mathrm{LL}^{-1}$.

\section{DISCUSSÃO}

O resultado encontrado nesse trabalho é coerente com os dados da literatura sobre atividade moluscicida de $E$. milii para gastrópodes aquáticos como Biomphalaria spp verificados em laboratório e também em condições de campo controladas e em condições naturais (Vasconcellos \& Schall, 1986; Baptista et al., 1992; Mendes et al., 1992; Mendes et al., 1997).

Já a ausência de atividade moluscicida para E. cotinifolia e E. tirucalli sobre L. unilamellata. 
contrasta quando comparada com outros moluscos. A ausência de toxidez de $E$. tirucalli sobre $L$. unilamellata diferencia-se do resultado obtido por Jurberg et al. (1985) que obtiveram DL 90 igual a 85 ppm ao testar a atividade da diluição aquosa do látex dessa eufórbia sobre adultos de $B$. glabrata. No entanto, existem peculiaridades na ação moluscicida dessa espécie, uma vez que o látex quando proveniente de espécimes de área sombreada não apresenta atividade (Jurberg et al., 1985). O espécime de $E$. tirucalli testado no presente estudo se encontra num local parcialmente sombreado, fato esse que pode explicar a ausência de atividade de toxidez. Em E. cotinifolia a diferença parece ser a presença de princípios ativos não hidrossolúveis justificando a ausência de efeito tóxico da diluição aquosa do látex dessa espécie vegetal sobre $L$. unilamellata.

Outra possibilidade para explicar a ausência de atividade moluscicida para $E$. cotinifolia e $E$. tirucalli é a variação na sensibilidade desses invertebrados as toxinas. Já foi constatada a existência de sensibilidade diferencial entre as espécies de Biomphalaria, tanto a toxinas de origem natural quanto de origem sintética (Jurberg et al., 1995; Sarquis et al., 1997; Bomfim et al., 2002).

Essa comparação sugere que diferenças químicas e/ou metabólicas entre as espécies de Euphorbia podem ser moduladas por fatores do ambiente, como a insolação, e ter diferentes impactos sobre a fisiologia da espécie de molusco testada. A análise desse conjunto de fatores pode nortear a seleção de plantas do gênero Euphorbia para estudos de atividade moluscicida, favorecer o delineamento dos ensaios experimentais e contribuir para a compreensão dos mecanismos tóxicos das substâncias envolvidas.

A atividade moluscicida em concentrações mais baixas do que em outras Euphorbiaceae, verificado para $E$. milii, é considerada vantagem (Vasconcellos \& Schall, 1986; Mendes et al., 1997) que pode ser verificada para o controle de $L$. unilamellata, uma vez que o látex de E. milii apresentou ação efetiva sobre esse gastrópode.

As reações observadas em $L$. unilamellata como resposta à aplicação do látex diluído de $E$. milii também aparecem em moluscos terrestres tratados com diferentes concentrações do extrato de semente de Thevetia peruviana (Panigrahi \& Raut, 1994) e frente a moluscicidas sintéticos (Paulini, 1959). Portanto tais reações podem ser típicas desses animais quando expostos à substâncias tóxicas.

\section{CONCLUSÃO}

Dentre as três espécies vegetais testadas apenas E. milii splendens exibiu atividade moluscicida elevada sobre L. unilamellata, e essa atividade em concentrações baixas sugere a presença de substâncias moluscicidas hidrossolúveis no látex.

A ausência de atividade moluscicida dos látices diluídos de $E$. cotinifolia e E. tirucalli sobre $L$. unilamellata, somada a toxidez dessas plantas para espécies de Biomphalaria, sugere a ocorrência de susceptibilidade diferencial entre gastrópodes pulmonados aquáticos e terrestres.

O látex de $E$. milii splendens pode vir a se constituir em estratégia de controle químico de moluscos terrestres.

\section{REFERÊNCIA}

ALFONSO, M. et al. Plantas molusquicidas de Cuba y su utilización. Revista de Protección Vegetal, v.15, n.2, p.118-24, 2000.

ALICATA, J.E. The life cicle of Postharmostomum gallinum, the cecal fluke of poultry. The Journal of Parasitology, v.26, n.2, p.135-43, 1940.

AL-ZANBAGI, N.A.; BARRET, J.; BANAJA, A.A. Laboratory evaluation of the molluscicidal properties of some SaudiArabian Euphorbiales against Biomphalaria pfeifferi. Acta Tropica, v.78, p.23-9, 2001.

BAPTISTA, D.F. et al. Evaluation of the molluscicidal property of Euphorbia splendens var. hislopii (N.E.B.) (Euphorbiaceae) - 2 - Investigation in lotic habitat. Memórias do Instituto Oswaldo Cruz, v.87, n.4, p.54953, 1992.

BESSA, E.C.A.; ARAÚJO, J.L.B. Ocorrência de autofecundação em Subulina octona (Bruguiére) (Pulmonata, Subulinidae) sob condições de laboratório. Revista Brasileira de Zoologia, v.12, n.3, p.719-23, 1995.

BOMFIM, T.C.B. et al. Toxidez seletiva do extrato etanólico bruto de Capsicum baccatum L. sobre Biomphalaria glabrata Say e Biomphalaria tenagophila Orbigny. Revista Brasileira de Plantas Medicinais, v.4, n.2, p.559, 2002.

BROWN, D.S. Pulmonate molluscs as intermediate hosts for digenetic trematodes. In: FRETTER, D.V.; PEAKE, J. (ed.). Pulmonates. London: Academic Publishers, 1978. v.2A, p.287-334.

CLARK, T.L.; APPLETON, C.C.; KUALSVIG, J.D. Schistosomiasis and the use of indigenous plant molluscicides: a rural South African perspective. Acta Tropica, v.66, p.93-107, 1997.

DUARTE, M.J.F. O ciclo evolutivo de Postharmostomun gallinum Witenberg, 1923, no estado do Rio de Janeiro, Brasil (Trematoda, Brachylaemidae). Revista Brasileira de Biologia, v.40, n.4, p.793-809, 1980.

DUTRA, A V.C. Aspectos da ecologia e da reprodução de Leptinaria unilamellata (ORBIGNY, 1835) (Gastropoda, Subulinidae). Revista Brasileira de Zoologia, v.5, n.4, p.581-91, 1988.

EL-KHOBI, T.A.G. Schistosomiasis control in a primary health care system. Memórias do Instituto Oswaldo Cruz, v.90, n.2, p.303-6,1995.

FRETTER, D.V.; PEAKE, J. Pulmonates. London: Academic Publishers, 1978. v.2A, 540p.

JURBERG, P.; CABRAL NETO, J.B.; SCHALL, V.T. Molluscicide activity of the "avelós" plant (Euphorbia

Rev. Bras. PI. Med., Botucatu, v.12, n.1, p.90-95, 2010. 
tirucalli L.) on Biomphalaria glabrata, the mollusc vector of schistoasomiasis. Memórias do Instituto Oswaldo Cruz, v.80, n.4, p.423-7, 1985.

JURBERG, P. Why it is difficult to control Biomphalaria glabrata, the vector snail of schistosomiasis. Memórias do Instituto Oswaldo Cruz, v.82, supl.6, p.203-7, 1987. JURBERG, P.; VASCONCELLOS, M.C.; MENDES, N.M. Plantas empregadas como moluscicidas: uma visão crítica. Memórias do Instituto Oswaldo Cruz, v.84, n.1, p.76-83,1989.

JURBERG, P. et al. Effect of Niclosamide (Bayluscide WP $70^{\mathrm{R}}$ ), Anacardium occidentale hexane extract and Euphorbia splendens latex on behaviour of Biomphalaria glabrata (Say, 1818), under laboratory conditions. Memórias do Instituto Oswaldo Cruz, v.90, n.2, p.191-4, 1995

LIU, S.Y. et al. Anthraquinones in Rheum palmatum and Rumex dentatus (Polygonaceae) and phorbol esters in Jatropha curcas (Euphorbiaceae) with molluscicidal activity against the schistosome vector snail Oncomelania hupensis. Tropical Medicine and International Health, v.2, n.2, p.179-88, 1997.

MABBERLEY, D.J. The plant-book: a portable dictionary of the higher plants. 2.ed. Cambridge: Cambridge University Press, 1997. 858p.

MALEK, E.A.; CHENG. T.C. Control of economically and medically important snails. In: Medical and Economic Malacology. New York: Academic Press, 1974. p.290-9. MARSTON, A.; HOSTETTTMANN, K. Plant molluscicides. Phytochemistry, v.24, n.4, p.639-52, 1985.

MENDES, N.M. et al. Evaluation of the molluscicidal properties of Euphorbia splendens var. hislopii (N.E.B.) (Euphorbiaceae).1- Experimental test in a lentic habitat. Memórias do Instituto Oswaldo Cruz, v.87, n.1, p.213,1992.

MENDES N.M. et al. Evaluation of the molluscicidal properties of Euphorbia splendens var. hislopii (N.E.B.) latex: experimental test in an endemic area in the estate of Minas Gerais, Brazil. Memórias do Instituto Oswaldo Cruz, v.92, n.5, p.719-24, 1997.

MICHELSON, E.H. The intermediate snail host: An agenda for future study. Memórias do Instituto Oswaldo Cruz, v.82, supl.4, p.1193-5, 1987.

OLIVEIRA, F.; AKISUE, G. Fundamentos de Farmacobotânica. 2.ed. São Paulo: Atheneu, 1997. 178p. OLIVEIRA, M.P.; VIEIRA, I.; OLIVEIRA, M.H.R. Comunicação no 1. Criação de moluscos em terrários e aquários (uma experiência em laboratório). Juiz de
Fora: Esdeva,1968. 15p.

PANIGRAHI, A.; RAUT, S.K. On the safe use of pesticides in controlling the terrestrial mollusc pests. Memórias do Instituto Oswaldo Cruz, v.88, n.2, p.293 -7, 1993.

PANIGRAHI, A.; RAUT, S.K. Thevetia peruviana (Family: Apocinaceae) in the control of slug and snail pests. Memórias do Instituto Oswaldo Cruz, v.89, n.2, p.24750, 1994.

PAULINI, E. Moluscicidas e outros métodos profiláticos. Revista Brasileira de Malariologia e Doenças Tropicais, v.11, p.595-623,1959.

PEREIRA, J.P.; SOUZA, C.P.; MENDES, N.M. Propriedades moluscicidas da Euphorbia cotinifolia L. Revista Brasileira de Pesquisas Médicas e Biológicas, v.11, n.6, p.345-51,1978.

RAUT, S.K.; PANIGRAHI, A. Feeding rhythm in the garden slug Laevicaulis alte (Soleolifera: Veronicellidae). Malacological Review, v.23, p.39-46, 1990.

RUG, M.; RUPPEL, A. Toxic activities of the plant Jatropa curcas against intermediate snail hosts and larvae of schistosomes. Tropical Medicine \& International Health, v.5, n.6, p.423-30, 2000.

SARQUIS, O.; PIERI, O.S.; SANTOS, J.A.A. Effects of bayluscide WP $70^{R}$ on the survival and water-leaving behaviour of Biomphalaria straminea, snail hosts of schistosomiasis in northeast Brazil. Memórias do Instituto Oswaldo Cruz, v.92, n.5, p.619-23, 1997.

STURROCK, R.F. Current concepts of snail control. Memórias do Instituto Oswaldo Cruz, v.90, n.2, p.2418,1995.

STURROCK, R.F. Schistosomiasis epidemiology and control: How did we get here and where should we go? Memórias do Instituto Oswaldo Cruz, v.96, p.17-27, 2001. THOMAS, J.D. The snail hosts of schistosomiasis: Some evolutionary and ecological perspectives in relation to control. Memórias do Instituto Oswaldo Cruz, v.90, n.2, p.195-204,1995.

VASCONCELLOS, M.C.; SCHALL, V.T. Látex of "coroa de cristo" (Euphorbia splendens): An effective molluscicide. Memórias do Instituto Oswaldo Cruz, v.81, n.4, p.475-6, 1986.

ZANI, C.L. et al. Molluscicidal miliamines from Euphorbia milii var. hislopii. Phytochemistry, v.34, n.1, p.89-95, 1993. YUAN, H.C. Schistosomiasis control in China. Memórias do Instituto Oswaldo Cruz, v.90, n.2, p.297-301, 1995. WORLD HEALTH ORGANIZATION-WHO. Molluscicidal screening and evaluation. Bulletin of the World Health Organization, v.33, 1965. 567p. 